\title{
Compromised Gut Associated Lymphoid Tissue is a Risk Factor for Postoperative Septic Complications in HIV-Seropositive Trauma Patients
}

\author{
Martin Mauser ${ }^{1} \cdot$ Deirdré Kruger $^{2} \cdot$ Sugeshnee Pather $^{3} \cdot$ Frank Plani $^{4}$
}

Accepted: 27 November 2020/Published online: 12 January 2021

(C) Société Internationale de Chirurgie 2021

\begin{abstract}
Background The gut associated lymphoid tissue (GALT) is an important part of the immune system and compromised in HIV treatment-naïve as well as in HIV-seropositive patients on antiretroviral treatment (ART) due to HIVinduced changes. The influence of the impaired GALT on the postoperative complication rate after surgery for penetrating abdominal trauma has not been investigated and the hypothesis that the HIV-induced changes of the GALT contribute to septic complications postoperatively was tested.

Material and methods This prospective study included patients who required a small bowel resection due to abdominal gunshot wounds. A bowel specimen was obtained in the index operation, and the T-lymphocytic quantity in the specimen was analyzed via immunohistochemistry to scrutinize whether these lymphocyte numbers had an impact on the postoperative outcome. Septic and postoperative complications were documented during the inhospital course and the first month after discharge.

Results In total, 62 patients were included in the study of which 38 patients were HIV-seronegative and 24 were HIV-seropositive. HIV-seropositive patients had a significantly lower quantity of CD4 $+\mathrm{T}$ cells in the GALT compared to the HIV-seronegative patients $(p=0.0001)$, which was also associated with a significantly higher rate of septic complications in the postoperative course. In the HIV-seropositive group, no significant differences were detected for T-lymphocytic quantity in the GALT between the HIV-treatment naïve and antiretroviral treatment groups.

Conclusion The compromised GALT in HIV-seropositive patients may predispose these patients to postoperative septic complications. Antiretroviral therapy does not result in an adequate immune reconstitution in this tissue.
\end{abstract}

Martin Mauser

mtmauser@gmx.de

Deirdré Kruger

deirdre.kruger@wits.ac.za

Sugeshnee Pather

sugeshnee.pather@nhls.ac.za

Frank Plani

frankplani@icloud.com

1 Department of Surgery, Faculty of Health Sciences, Chris Hani Baragwanath Academic Hospital, University of the Witwatersrand, York Road, Johannesburg, Parktown, Gauteng, South Africa
2 Department of Surgery, Faculty of Health Sciences, University of the Witwatersrand, York Road, Johannesburg, Parktown, Gauteng, South Africa

3 National Health Laboratory Service, Chris Hani Baragwanath Academic Hospital, Faculty of Health Sciences, School of Pathology, University of the Witwatersrand, Johannesburg, Parktown, Gauteng, South Africa

4 Chris Hani Baragwanath Academic Hospital, Faculty of Health Sciences, University of the Witwatersrand, Johannesburg, Parktown, Gauteng, South Africa 


\section{Introduction}

Acquired immunodeficiency syndrome (AIDS)-related pathologies requiring surgery have decreased markedly since the introduction of combined antiretroviral therapy (ART) [1]. Reconstitution of the immune system after starting antiretrovirals (ARVs) resulted in an increased life expectancy [2] and reduced the complication rate after surgical procedures compared to the pre-ART period [3].

However, a considerable number of HIV-seropositive patients on ARVs only show an incomplete immune recovery at the time of surgical intervention [4]. Furthermore, problems are encountered in HIV-seropositive patients requiring urgent surgery who are newly diagnosed or those who have defaulted their treatment, the latter of which is often encountered among trauma patients [5].

Current research on HIV-related changes of the gut associated lymphoid tissue (GALT) has documented a more severe decrease in CD4 $+\mathrm{T}$ cells compared to the peripheral blood [6]. Weakening of the local immune system due to depletion of CD4 $+\mathrm{T}$ cells in the lamina propria together with structural changes of the bowel seems to predispose HIV-seropositive patients to bacterial translocation [7].

This prospective study compared the postoperative course between HIV-seropositive and HIV-seronegative patients who sustained penetrating abdominal trauma and determined whether the T-lymphocytic quantity of the GALT impact on the outcome.

We hypothesized that the HIV-induced changes of the GALT contribute to septic complications postoperatively.

\section{Material and methods}

In this prospective study, we recruited HIV-seropositive and HIV-seronegative adult patients from December 2018 to March 2020 at the Chris Hani Baragwanath Academic Hospital (CHBAH) Trauma Unit in Johannesburg, South Africa, who required surgery for abdominal gunshot injuries with bowel involvement. Exclusion criteria were uncontrolled diabetes mellitus (A1C level $>7 \%$ ), active tuberculosis infection, a history of prolonged steroid use, pre-existing organ failure and demise within $72 \mathrm{~h}$ of admission. The data collection was done independently and prospectively by the trauma research group of CHBAH.

For comparison of the bowel specimen, a control group of eleven patients was recruited. These patients underwent small bowel resection for non-traumatic indications, without physiological derangements or signs of sepsis at the time of surgery and had all a confirmed HIV-negative serostatus. For the distal small bowel specimens, patients who underwent stoma reversal with practically no comorbidities were recruited. For the specimens of the proximal bowel, we recruited three patients who had surgery for malignancies.

The protocol was approved by the Human Research Ethics Committee (Medical) of the University of the Witwatersrand (clearance number M180914). Informed consent was obtained from all patients, or if the patient had a decreased level of consciousness on admission, consent was signed by the patient's next of kin.

A full thickness, non-contused specimen of the small intestine, at least $5 \mathrm{~mm}$ in size, was obtained by the surgical team during index laparotomy from the segment of the bowel requiring resection due to the sustained injuries without knowing the patient's confirmed HIV status yet. The entirety of the sustained intra-abdominal injuries was graded according to the penetrating abdominal trauma index (PATI) [8] which is commonly used and validated scoring system for these injuries [9].

HIV status and CD4 + T-lymphocyte count in the peripheral blood were tested in all patients. In HIVseropositive patients, viral load, ARV treatment status, treatment duration, drug combinations and presence of AIDS-defining conditions were documented.

The follow-up of the study patients was restricted to inhospital course and a weekly review in our trauma clinic for the period of one month after discharge from the hospital. The outcome was measured according to the number and nature of septic complications, as well as postoperative surgical complications, which were classified according to the Clavien-Dindo classification [10]. Septic complications included superficial and deep surgical site infections, septic intra-abdominal collections (diagnosed via radiological imaging or during relook), septicaemia with positive blood cultures, fungemia, pneumonia and urinary tract infections. Postoperative complications according to the ClavienDindo classification of grades 1-2 were categorized as minor, whereas grades 3-5 were major complications. The latter division was based on grade 3 complications and higher needing invasive interventions, either surgically, endoscopically or radiologically.

\section{Laboratory investigations of bowel tissue}

Immunohistochemistry (IHC) was performed on all bowel specimens by the National Health Laboratory Service's Division of Histopathology at CHBAH. CONFIRM antiCD4 (SP35) rabbit monoclonal primary antibody and CONFIRM anti-CD8 (SP57) rabbit monoclonal primary antibody (Roche/Ventana Medical Systems, Inc.; Tucson, Arizona) were used for IHC staining. The antibodies were 
stored at $2-8{ }^{\circ} \mathrm{C}$ until used in the detection kits specific for the Ventana BenchMark XT automated slide stainer.

Light microscopic examination was performed independently by the principle investigator (MM) and a histopathologist in a blinded manner without any accompanying clinical information.

The number of CD4 + and CD8 + lymphocytes of the lamina propria, hereafter referred to as lamina propria lymphocytes (LPL), was assessed at X400 magnification within 10 non-overlapping high-power fields by manual counting with the aid of Zeiss (Zeiss Inc.; Jena, Germany) Axioscope and software and expressed as number of cells per $\mathrm{mm}^{2}$.

\section{Statistical analysis}

The chi-squared test or Fisher's exact test was used, as appropriate, to assess the relationships between categorical variables according to the patients' HIV status.

The relationship between the continuous variables and HIV status was assessed by the t-test. Where the data did not meet the assumptions of these tests, a non-parametric alternative, the Wilcoxon rank sum test was used. Sample size calculations were carried out in $\mathrm{G}^{*}$ Power, and a minimum sample size of 54 patients was required for determination of a large size effect [11].

Data analysis was carried out using STATA version 15.1 for Windows. $P$-values $<0.05$ were considered significant.

\section{Results}

\section{Patient recruitment, demographics and perioperative factors}

During the study period, 167 patients required a laparotomy for abdominal gunshot wounds, of which 93 patients were excluded because they had no small bowel injuries, and 12 were excluded due to demise within $72 \mathrm{~h}$ of admission. In total, 62 patients were recruited. The demographics, injury severity, other relevant perioperative factors in the study population, as well as the injured organs, are listed in Table 1. The HIV-seropositive patients were significantly older than the HIV-seronegative patients.

Of the $24 \mathrm{HIV}$-seropositive patients, 12 patients were newly diagnosed, while 12 patients knew their HIV status. Of those who knew their HIV status, 10 (83\%) were taking ARVs. The mean $( \pm \mathrm{SD})$ treatment duration in the latter group was 5.5 (4.1) years. The median CD4 + T-lymphocyte count of the entire HIV-seropositive study population on admission was 338 cells/ $\mu$ l (IQR 204-471). The median viral load was 8010 copies/ml (IQR 368-64 450) which is equivalent to a mean $( \pm \mathrm{SD})$ of 3.7 (1.6) $\mathrm{Log}$ copies/ml. No significant difference in the CD4 + T-lymphocyte count between HIV naïve and patients on ARVs was detected in the admission blood of the HIV-seropositive patients (HIV naïve: 320; IQR 196-440 vs. HIV treatment: 390; IQR 238-482, $p=0.58$; data not shown). Only two HIV-seropositive patients showed clear signs of AIDS-defining conditions, both of them were treatmentnaïve.

The amount of blood products, including red blood cells, fresh frozen plasma and platelets did not differ between the groups.

\section{Comparison of lymphocyte quantity in the GALT in relation to $\mathrm{HIV}$ infection and treatment}

The lamina propria of the HIV-seropositive patients showed a severe depletion of $\mathrm{CD} 4+\mathrm{T}$ cells, with the median number (IQR) of CD4 + LPL significantly lower compared to the HIV-seronegative patients at 252 (176-413) cells $/ \mathrm{mm}^{2}$ vs. 905 (813-997) cells $/ \mathrm{mm}^{2}$, respectively $(p=0.0001)$. Conversely, the quantity of CD8 + LPL was significantly higher in HIV-seropositive (1 143; IQR 826-1 235 cells $/ \mathrm{mm}^{2}$ ) vs. HIV-seronegative (512; IQR 410-572 cells $\left./ \mathrm{mm}^{2}\right)$ patients $(p=0.0001)$. In the HIV-seropositive patient group, a significantly lower CD4 + LPL count and a significantly higher CD8 + LPL count were associated with a significantly higher rate of septic complications compared to the HIV-seronegative patients $(p=0.002)$; data shown in Fig. 1 and Table 2.

Within the group of HIV-seropositive patients, there was no significant difference between the treatment-naive and the ARV-treated patients in the number of the CD4 + LPL [treatment-naïve 229 (IQR 166-260) vs. treated 349 (IQR 225-425) cells $/ \mathrm{mm}^{2} ; p=0.18$ ] and CD8 + LPL [treatment-naïve 1175 (IQR 878-1 307) vs. treated 979 (IQR 778-1 231) cells/mm $\mathrm{mm}^{2} ; p=0.27$; data not shown].

The inclusion and analysis of the non-trauma, HIVseronegative control group provides reference values for CD4 + LPL and CD8 + LPL. These reference values are as follows [median (IQR)]: CD4 + LPL: 1121 (720-1 199); and CD8 + LPL: 414 (371-612) cells $/ \mathrm{mm}^{2}$.

Figure 2 displays microscopic images following IHC and shows the difference of the CD4 + LPL between a treatment-naïve HIV-seropositive patient (Fig. 2a) and a HIV-seronegative patient (Fig. 2b), with CD4 $+\mathrm{T}$ cells embrowned.

\section{Clinical course and complications}

In the postoperative follow-up of the study patients, significantly more patients with septic complications 
Table 1 Demographics, perioperative factors and injured organs of study patients according to HIV-serostatus

\begin{tabular}{|c|c|c|c|}
\hline Variable & HIV-seronegative patients $(n=38)$ & HIV-seropositive patients $(n=24)$ & $P$ value \\
\hline Age, years & $31(25-40)$ & $41(32-47)$ & 0.013 \\
\hline \multicolumn{4}{|l|}{ Gender } \\
\hline Male & $36(95 \%)$ & $22(92 \%)$ & 0.64 \\
\hline Female & $2(5 \%)$ & $2(8 \%)$ & \\
\hline Time injury-to-operation $(h)$ & $4(3-6)$ & $5(4-6)$ & 0.51 \\
\hline PATI & $27(17-33)$ & $27(16-32)$ & 0.79 \\
\hline $\mathrm{pH}$, on admission & $7,34(7,26-7,43)$ & $7,33(7,26-7,36)$ & 0.32 \\
\hline Lactate $(\mathrm{mmol} / \mathrm{L})$, on admission & $4,5(3,2-6,6)$ & $4,7(3,1-5,4)$ & 0.82 \\
\hline Bicarb (mmol/L), on admission & $21,7(16,3-23,2)$ & $21,9(17,8-23,8)$ & 0.59 \\
\hline $\mathrm{BE}(\mathrm{mmol} / \mathrm{L})$, on admission & $-4,1(-8,8$ to $-1,0)$ & $-4,0(-7,4$ to $-1,6)$ & 0.85 \\
\hline \multicolumn{4}{|l|}{ Fecal contamination } \\
\hline Mild & $5(13 \%)$ & $3(13 \%)$ & \\
\hline Moderate & $10(26 \%)$ & $6(25 \%)$ & $>0.99$ \\
\hline Severe & $23(61 \%)$ & $15(63 \%)$ & \\
\hline Colonic injury & $25(66 \%)$ & $13(54 \%)$ & 0.43 \\
\hline Liver injury & $3(8 \%)$ & $4(17 \%)$ & 0.42 \\
\hline Stomach injury & $5(13 \%)$ & $5(21 \%)$ & 0.49 \\
\hline Vascular injury & $11(29 \%)$ & $4(17 \%)$ & 0.37 \\
\hline Involvement of other organs & $10(26 \%)$ & $9(38 \%)$ & 0.40 \\
\hline Inotrope requirement & $23(61 \%)$ & $15(63 \%)$ & $>0.99$ \\
\hline Inotrope duration $(h)$ & $41(7-66)$ & $50(24-96)$ & 0.21 \\
\hline Hypotension (SBP $<90 \mathrm{mmHg}$ ), on admission & $10(26 \%)$ & $7(29 \%)$ & $>0.99$ \\
\hline Damage control surgery & $19(50 \%)$ & $12(50 \%)$ & $>0.99$ \\
\hline
\end{tabular}

Continuous numerical variables presented as median (interquartile range [IQR]). Categorical variables expressed as absolute and relative frequencies (in brackets)

$R B C$ red blood cell; FFP fresh frozen plasma; $S B P$ systolic blood pressure

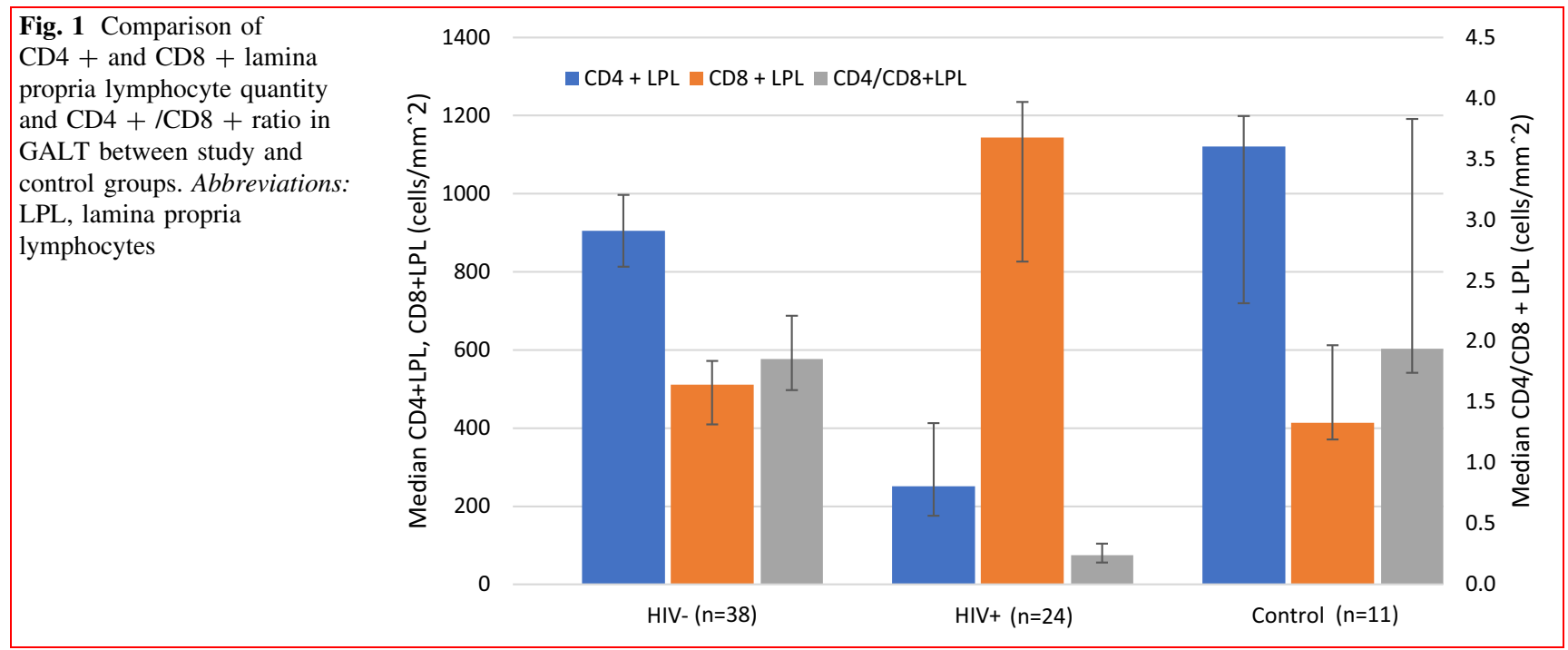

( $p=0.002$; Table 3 ) were observed in the HIV-seropositive group compared to the HIV-seronegative group. The higher complication rate was primarily due to superficial surgical site infections that were significantly more common in the HIV-seropositive patients $(p=0.005$; Table 3). HIV-seropositive patients had an anastomotic leak rate of $38 \%$, HIV-seronegative patients leaked in $18 \%$ of the cases; this difference did not reach statistical 
Table 2 Comparison of quantity of CD4 + LPL and CD8 + LPL between HIV-seronegative and HIV-seropositive patients in relation to septic complications

\begin{tabular}{llll}
\hline & HIV-seronegative patients $(n=38)$ & HIV-seropositive patients $(n=24)$ & $p$ value \\
\hline CD4 + LPL (cells $\left./ \mathrm{mm}^{2}\right)$ & $905(813-997)$ & $252(176-413)$ & 0.0001 \\
CD8 + LPL (cells $\left./ \mathrm{mm}^{2}\right)$ & $512(410-572)$ & $1143(826-12235)$ & 0.0001 \\
Patients with septic complications & $20(53 \%)$ & $22(92 \%)$ & 0.002 \\
\hline
\end{tabular}

Continuous numerical variables presented as median (interquartile range [IQR]). Categorical variables expressed as absolute and relative frequencies (in brackets)

$L P L$ lamina propria lymphocytes; $\mathrm{mm}$ Millimeter

a

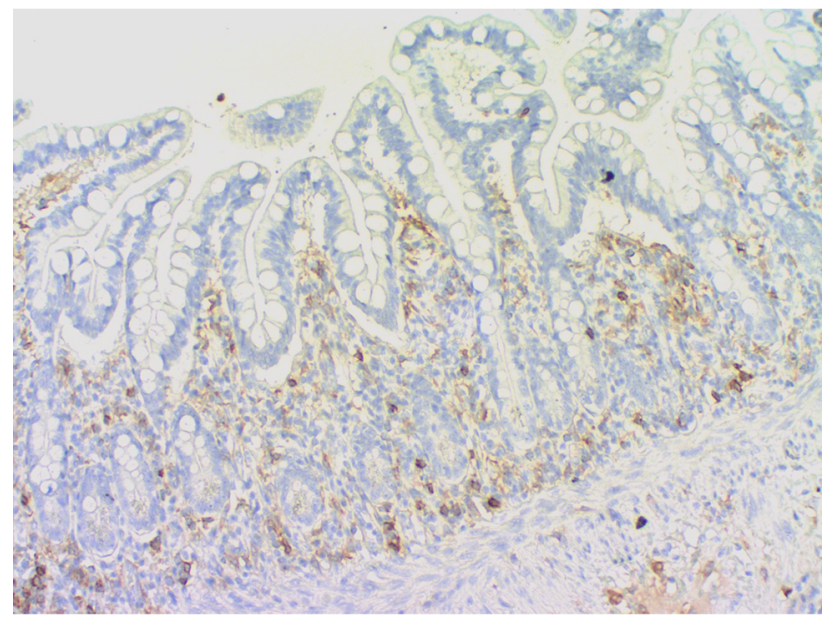

b

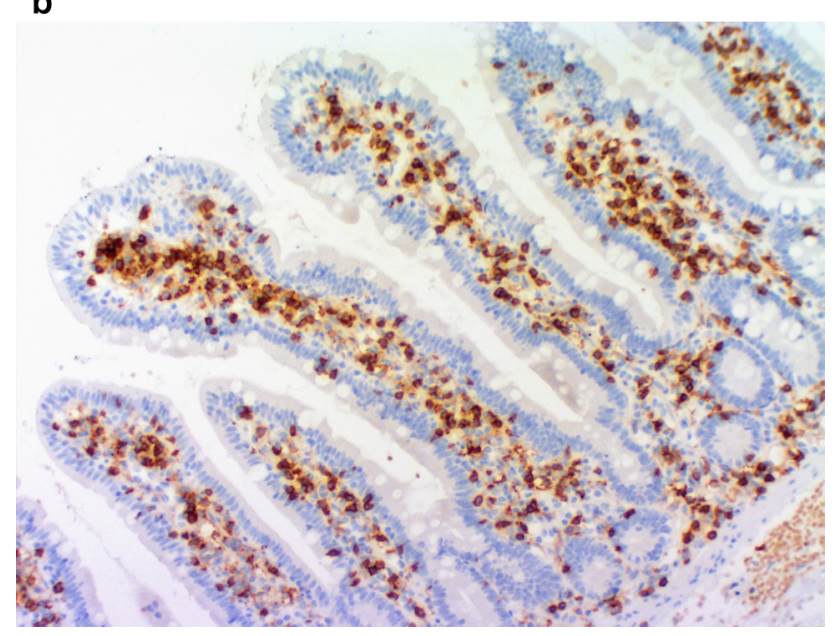

Fig. 2 Immunohistochemistry analysis of $\mathrm{CD} 4+\mathrm{T}$ cells in the lamina propria of a treatment-naïve HIV-seropositive patient $\mathbf{a}$ and a HIV-seronegative patient b. Specification: small bowel, $\times 100$ magnification

significance $(p=0.14)$. With regard to the time of diagnosing the anastomotic leak, the mean $( \pm \mathrm{SD})$ time to notice this complication was significantly later in the HIV- seropositive patients with 10.4 (1.4) days, compared to the HIV-seronegative patients [6.9 (1.6) days; $p=0.0003$ ].

A sub-analysis between the treatment-naïve HIVseropositive patients and patients on ARVs showed a similarly high complication rate in both groups, and the patients on ARVs did not have significantly fewer septic problems (Table 4).

\section{Discussion}

This study determined the T-lymphocytic quantity in the lamina propria of the GALT in the small bowel and investigated whether it has an influence on the postoperative course after penetrating abdominal trauma. Moreover, special emphasis was to investigate whether HIV-infection, and specifically, preexisting HIV-induced changes of the GALT had an impact on postoperative septic complications.

The demographic profile, perioperative factors, injury pattern and severity showed no significant differences between HIV-seropositive and HIV-seronegative patients, hence the groups were well matched, except for a significant difference in age between the two groups.

In the analysis of the GALT, we observed that HIVseropositive patients had significantly less CD4 $+\mathrm{T}$ cells and significantly more CD8 $+\mathrm{T}$ cells in the LP compared to the HIV-seronegative study patients and the non-trauma, HIV-seronegative control group. Lower CD4 $+\mathrm{T}$ cells and higher $\mathrm{CD} 8+\mathrm{T}$ cells were also associated with an increased rate of septic complications in this study population.

Our finding reflects Brenchley et al.'s observation in which he described a severe depletion of the mucosal $\mathrm{CD} 4+\mathrm{T}$ cells after primary HIV-infection that persists for the further course of disease resulting in a severe lymphopenia [7]. The cause for this massive decrease in CD4 + LPL is still debatable and is most likely multifactorial; virus-induced Fas/FasL-mediated apoptosis, virus triggered cytolysis, lymphocyte destruction by cytotoxic 
Table 3 Comparison of clinical course and complications according to HIV-serostatus

\begin{tabular}{|c|c|c|c|}
\hline Parameter & HIV-seronegative patients $(n=38)$ & HIV-seropositive patients $(n=24)$ & $P$ value* \\
\hline Septic complications & $20(53 \%)$ & $22(92 \%)$ & 0.002 \\
\hline Superficial SSI & $17(45 \%)$ & $18(75 \%)$ & 0.005 \\
\hline Deep SSI & $8(21 \%)$ & $8(34 \%)$ & 0.22 \\
\hline Septicemia (positive BC) & $14(37 \%)$ & $14(58 \%)$ & 0.28 \\
\hline \multicolumn{4}{|l|}{ Pneumonia } \\
\hline No pneumonia & $29(76 \%)$ & $16(67 \%)$ & \multirow{3}{*}{0.13} \\
\hline VAP & $5(13 \%)$ & $5(21 \%)$ & \\
\hline Postop pneumonia (not ventilator-associated) & $4(11 \%)$ & $3(13 \%)$ & \\
\hline Septic intra-abdominal collection & $16(42 \%)$ & $14(58 \%)$ & 0.30 \\
\hline Anastomotic leak & $7(18 \%)$ & $9(38 \%)$ & 0.14 \\
\hline Repair break down & $3(8 \%)$ & $3(13 \%)$ & 0.66 \\
\hline Number of relooks & $1(0-3)$ & $1(0-4)$ & 0.40 \\
\hline ICU-LOS (in days, censored for death) & 30 (15-ne) & $18(5-33)$ & 0.78 \\
\hline Ventilatory days & $6(3-15)$ & $4(4-18)$ & 0.93 \\
\hline \multicolumn{4}{|l|}{ Renal failure } \\
\hline Dialysis required & $5(13 \%)$ & $4(17 \%)$ & \multirow[t]{2}{*}{0.67} \\
\hline Dialysis not required & $5(13 \%)$ & $1(4 \%)$ & \\
\hline \multicolumn{4}{|l|}{ Clavien-Dindo classification } \\
\hline $0-2$, minor & $19(50 \%)$ & $7(29 \%)$ & \multirow[t]{2}{*}{0.12} \\
\hline $3-5$, major & $19(50 \%)$ & $17(71 \%)$ & \\
\hline Mortality & $7(18 \%)$ & $9(38 \%)$ & 0.14 \\
\hline
\end{tabular}

*Bold values are statistically significant. Continuous numerical variables presented as median (interquartile range [IQR]). Categorical variables expressed as absolute and relative frequencies (in brackets)

$B C$ blood culture; ICU-LOS intensive care unit length of stay; SSI surgical site infections; VAP ventilator-associated pneumonia; ne not estimable

Table 4 Sub-analysis for septic and surgical complications of HIV-seropositive patients according to treatment status

\begin{tabular}{lll}
\hline & HIV-seropositive naïve $(n=14)$ & HIV-seropositive on treatment $(n=10)$ \\
\hline $\begin{array}{ll}\text { Number of septic complications } \\
0\end{array}$ & $0(0 \%)$ & $2(20 \%)$ \\
1 or more & $14(100 \%)$ & $8(80 \%)$ \\
Clavien-Dindo classification & \\
$0-2$ minor & $4(29 \%)$ & $3(30 \%)$ \\
$3-5$ major & $10(71 \%)$ & $7(70 \%)$ \\
\hline
\end{tabular}

Categorical variables expressed as absolute and relative frequencies (in brackets)

T-lymphocytes and NK-cells as well as activation-induced cell death of lymphocytes seem to play a role [12].

Among the HIV-infected patients in our study, we did not find a significant quantitative difference of $\mathrm{CD} 4+\mathrm{T}$ cells in the GALT of treatment-naïve patients and patients on ARVs. The patients on treatment showed only a slightly higher CD4 $+\mathrm{T}$ cell count on average, indicating a partial recovery, but their numbers fell far short of the HIV- negative study members, which confirms results of current HIV research [13].

We observed a significantly higher quantity of CD8 $+\mathrm{T}$ cells in the LPL of the HIV-seropositive patients compared to the HIV-seronegative patients. Despite the observation that $\mathrm{CD} 8+\mathrm{T}$ cell-induced responses are essential for viral control and limit the viral replication in the acute and chronic disease phase [14, 15], the accumulation of activated $\mathrm{CD} 8+\mathrm{T}$ cells in the $\mathrm{LP}$ also seems to have a 
negative effect on the reexpansion of the $\mathrm{CD} 4+\mathrm{T}$ cell pool $[13,16]$.

In line with the findings of the CD4 + cell count in the HIV-seropositive patients, we also did not detect a significant difference in the $\mathrm{CD} 8+\mathrm{T}$ cell count between treatment-naïve and treated patients, showing again the insufficient efficacy of the ARVs in restoration of lymphocytes in the LP of the GALT, a finding broadly supported by current HIV research [17-19].

Significantly more patients in the HIV-seropositive group suffered septic complications, especially superficial surgical site infections played a major role. More than a third of the HIV-infected patients was affected by an anastomotic leak which is an unacceptably high number and makes intrinsic, systemic causes, such as an impaired GALT, likely.

Fascial as well as anastomotic healing is reparative processes involving fibroblasts that are essential for remodeling the extracellular matrix and collagen synthesis. The fibroblast function is regulated by cytokines derived from monocytes/macrophages as well as from CD4 + Tlymphocytes [20]. The severe depletion of T-lymphocytes in the GALT may result in an altered fibroblast activity which affects the collagen and proteoglycan synthesis and influences wound strength and resilience. The latter is further supported by the finding that patients who suffered from an anastomotic leak had a significant lower CD4 $+\mathrm{T}$ cell count in their GALT compared to patients without a leak. The observation of impaired wound- and anastomotic healing is further supported by a rat model showing that a severe decrease in $\mathrm{CD} 4+\mathrm{T}$ cells resulted in weaker wounds measured with several mechanical properties [21].

The analysis of the T-lymphocytic quantity of the GALT revealed a significant association between a decreased quantity of CD4 + LPL and more septic postoperative complications. The loss of these immune cells in the GALT may therefore be a predisposing factor for propagation of enteric bacteria into the blood stream with subsequent septic events. Supporting this presumption is the impressive mouse model from Gautreaux et al. in which $\mathrm{T}$ cell depletion resulted in a significantly higher translocation of E. coli from the small bowel [22].

Identically low CD4 $+\mathrm{T}$ cell quantity in the GALT was documented as a common denominator in treatment-naïve as well as treated HIV-seropositive patients. There is no doubt about the beneficial role that ARVs have in the treatment and prognosis of HIV-infected patients. However, the very limited efficacy on the reconstitution of the GALT may be the "Achilles heel" in the perioperative phase. Several reports about the influence of HIV on surgical treatment documented that the treatment with ARVs did not result in better outcomes or less complications which highlights the significance of this problem [23-25].
There is a paucity of data regarding the impact of age on the GALT. Quantity of LPL does not seem to be affected, but functional changes, especially decreased Interleukin-2 production, was observed in higher age groups [26] which, in turn, could have contributed to the higher rate of septic complications in the HIV-seropositive study group that was also significantly older.

Our research project was not without limitations. The number of 62 patients only had the statistical power to determine large size effects. Hence, more subtle significant associations might have been missed, not because there is no significant relationship but because there is insufficient evidence due to the small sample size. Consecutive patient recruitment resulted in groups that were not age-matched which could have led to biased results in the comparison between HIV-seropositive and HIV-seronegative patients.

Furthermore, the vast majority of our patients were male patients, and only four female patients were recruited. This gender imbalance makes it difficult to generalize our results as the new insights gained related almost exclusively to men.

No considerations were given to the nutritional status of the patients. It is documented that hypoalbuminemia is a risk factor for impaired wound and anastomotic healing [27], hence inclusion of this variable would have improved the study design.

In conclusion, we documented in HIV-seropositive patients a significantly higher septic complication rate compared to the HIV-seronegative patients which was primarily due to superficial surgical site infections. HIVseropositive patients had a significantly lower CD $4+\mathrm{T}$ cell quantity and a significantly higher $\mathrm{CD} 8+\mathrm{T}$ cell quantity in their GALT which may predispose them to postoperative septic complications. The alterations of the GALT in HIV-seropositive patients on ART were persistent and almost unchanged in terms of $\mathrm{CD} 4+\mathrm{T}$ cell recovery.

Acknowledgements The contribution(s) of the following people and institutions were instrumental in the realization of this project: The team of Division of Histopathology, NHLS at CHBAH, that provided immunohistochemistry specimens and the team from the Charlotte Maxeke Johannesburg Academic Hospital, National Health Laboratory Service flow cytometry laboratory, who provided an excellent and reliable service in the blood analysis.

Funding The study was sponsored by the trauma unit of the Chris Hani Baragwanath Academic Hospital.

\section{Compliance with ethical standards}

Conflict of interest The author declares that they have no conflict of interest.

Ethical Approval Throughout the study we adhered to the ethical requirements. 
Informed consent Informed consent was obtained from all individual participants included in the study.

\section{References}

1. Saltzman DJ, Williams RA, Gelfand DV et al (2005) The surgeon and AIDS. Arch Surg 140(10):961-967

2. Sabin CA (2013) Do people with HIV infection have a normal life expectancy in the era of combination antiretroviral therapy? BMC Med 11(1):1-7

3. Horberg MA, Hurley LB, Klein DB et al (2006) Surgical outcomes in human immunodeficiency virus-infected patients in the era of highly active antiretroviral therapy. Arch Surg 141(12):1238-1245

4. Chichom-Mefire A, Azabji-Kenfack M, Atashili J (2015) CD4 count is still a valid indicator of outcome in HIV-infected patients undergoing major abdominal surgery in the era of highly active antiretroviral therapy. World J Surg 39(7):1692-1699. https://doi. org/10.1007/s00268-015-2994-8

5. Wiseman SM, Forrest JI, Chan JE, Zhang W et al (2012) Factors predictive of 30 day postoperative mortality in HIV/AIDS patients in the era of highly active antiretroviral therapy. Ann Surg 256(1):170-176

6. Brenchley JM, Douek DC (2008) HIV infection and the gastrointestinal immune system. Mucosal Immunol 1(1):23-30

7. Brenchley JM, Price DA, Douek DC (2006) HIV disease: fallout from a mucosal catastrophe? Nat Immunol 7(3):235-240

8. Moore EE, Dunn EL, Moore JB et al (1981) Penetrating abdominal trauma index. J Trauma 21(6):439-445

9. Gomez-Leon JF (2004) Penetrating abdominal trauma index: sensitivity and specificity for morbidity and mortality by ROC analysis. Indian J Surg 66(6):347-351

10. Dindo D, Demartines N, Clavien PA (2004) Classification of surgical complications: a new proposal with evaluation in a cohort of 6336 patients and results of a survey. Ann Surg 240(2):205-213

11. Faul F, Erdfelder E, Lang AG, Buchner A (2007) G*Power 3: a flexible statistical power analysis program for the social, behavioral, and biomedical sciences. Behav Res Methods 39(2):175-191

12. Mehandru S (2007) The gastrointestinal tract in HIV-1 Infection: questions, answers, and more questions! PRN Noteb 12:88-98

13. Mehandru S, Poles MA, Tenner-Racz K et al (2006) Lack of mucosal immune reconstitution during prolonged treatment of acute and early HIV-1 infection. PLoS Med 3(12):e484

14. Pandrea I, Gaufin T, Gautam R et al (2011) Functional cure of SIVagm infection in rhesus macaques results in complete recovery of CD4+T cells and is reverted by $\mathrm{CD} 8+$ cell depletion. PLoS Pathog 7(8):e1002170
15. Streeck H, Lu R, Beckwith N et al (2014) Emergence of individual HIV-specific CD8 T Cell responses during primary HIV-1 Infection can determine long-term disease outcome. J Virol 88(21):12793-12801

16. Guadalupe M, Reay E, Sankaran S et al (2003) Severe CD4+ T-cell depletion in gut lymphoid tissue during primary human immunodeficiency virus type 1 infection and substantial delay in restoration following highly active antiretroviral therapy. J Virol 77(21):11708-11717

17. Pantaleo G, Demarest JF, Soudeyns H et al (1994) Major expansion of CD8 $\mathrm{T}$ cells with a predominant $\mathrm{V}$ beta usage during the primary immune response to HIV. Nature 370(6489):463-467

18. Guadalupe M, Sankaran S, George MD et al (2006) Viral suppression and immune restoration in the gastrointestinal mucosa of human immunodeficiency virus type 1 -infected patients initiating therapy during primary or chronic infection. J Virol 80(16):8236-8247

19. Shacklett BL, Cox CA, Sandberg JK et al (2003) Trafficking of human immunodeficiency virus type 1 -specific CD8+T cells to gut-associated lymphoid tissue during chronic infection. J Virol 77(10):5621-5631

20. Yamamura Y, Gupta R, Morita Y et al (2001) Effector function of resting T cells: activation of synovial fibroblasts. J Immunol 166(4):2270-2275

21. Davis PA, Corless DJ, Aspinall R et al (2001) Effect of CD4+ and $\mathrm{CD} 8+$ cell depletion on wound healing. $\mathrm{Br} \mathrm{J}$ Surg 88(2):298-304

22. Gautreaux MD, Deitch EA, Berg RD (1994) T lymphocytes in host defense against bacterial translocation from the gastrointestinal tract. Infect Immun 62(7):2874-2884

23. Deneve JL, Shantha JG, Page AJ et al (2010) CD4 count is predictive of outcome in HIV-positive patients undergoing abdominal operations. Am J Surg 200(6):694-699

24. Shanthamurthy D, Manesh A, Zacchaeus NG et al (2018) Perioperative outcomes in human immunodeficiency virus-infected patients-the PRO HIV study. Int J STD AIDS 29(10):968-973

25. Grubert TA, Reindell D, Kästner R et al (2002) Rates of postoperative complications among human immunodeficiency virusinfected women who have undergone obstetric and gynecologic surgical procedures. Clin Infect Dis 34(6):822-830

26. Beharka AA, Paiva S, Leka LS et al (2001) Effect of age on the gastrointestinal-associated mucosal immune response of humans. J Gerontol 56A(5):B218-B223

27. Mauser M, Bartsokas C, Brand M et al (2018) Postoperative CD4 counts predict anastomotic leaks in patients with penetrating abdominal trauma. Injury 50(1):167-172

Publisher's Note Springer Nature remains neutral with regard to jurisdictional claims in published maps and institutional affiliations. 